\title{
The Impact of 120 Minutes of Match-Play on Recovery and Subsequent Match Performance: A Case Report in Professional Soccer Players
}

\author{
Nathan Winder ${ }^{1}$, Mark Russell ${ }^{2}$, Robert J. Naughton ${ }^{3}$ and Liam D. Harper ${ }^{3, *}$ (D) \\ 1 Leeds United Football Club, Leeds LS23 7BA, UK; nathan.winder@leedsunited.com \\ 2 School of Social and Health Sciences, Leeds Trinity University, Leeds LS18 5HD, UK; \\ m.russell@leedstrinity.ac.uk \\ 3 School of Human and Health Sciences, University of Huddersfield, Huddersfield HD1 3DH, UK; \\ r.naughton@hud.ac.uk \\ * Correspondence: 1.harper@hud.ac.uk; Tel.: +44-(0)-14-8447-1157
}

Received: 24 January 2018; Accepted: 9 March 2018; Published: 13 March 2018

\begin{abstract}
The influence of a match including extra-time (ET) on subsequent 90 min match performance and recovery has not been investigated. Four professional soccer players played in three competitive matches in a 7-day period: matches one (MD1) and three (MD3) lasted 90 min and match 2 (MD2) lasted 120 min (i.e., included ET). Physical (total and high-intensity (HI) distance covered, accelerations and decelerations, and mechanical load) and technical performances (pass and dribble accuracy) were analyzed throughout match-play. Subjective measures of recovery and countermovement jump (CMJ) height were made 36-42 h post-match. Post-MD2, there were very or most likely harmful effects of ET on CMJ height ( $-6 \pm 9 \%)$, muscle soreness $(+18 \pm 12 \%)$, and fatigue $(+27 \pm 4 \%)$ scores, and overall wellness score $(-13 \pm 5 \%)$ compared to post-MD1. Furthermore, there were very likely harmful effects on muscle soreness $(+13 \pm 14 \%)$, wellness scores $(-8 \pm 10 \%)$, and CMJ height $(-6 \pm 9 \%)$ post-MD3 vs. post-MD1. There was a possibly harmful effect of ET on HI distance covered during MD3, along with reductions in pass (-9.3\%) and dribble (-12.4\%) accuracy. An ET match negatively impacted recovery $36 \mathrm{~h}$ post-match. Furthermore, in some players, indices of performance in a 90 min match played $64 \mathrm{~h}$ following ET were compromised, with subsequent recovery also adversely affected.
\end{abstract}

Keywords: football; accelerometry; fatigue

\section{Introduction}

The influence of $120 \mathrm{~min}$ of soccer match-play (i.e., matches requiring a $30 \mathrm{~min}$ extra-time period; ET), on physical and technical performance, and functional and biochemical measures of recovery has recently been investigated [1-4]. High-intensity distance covered and distance covered per minute reduces during the two 15 min periods of ET compared to other 15 min periods during competitive soccer match-play [3]. Furthermore, the number of passes and dribbles reduces during the last 15 min of ET compared to prior 15 min periods played during elite professional soccer matches [1]. Neuromuscular fatigue of central and peripheral origin develops during ET, which negatively impacts sprint performance and muscle force production [5], with reductions in muscle glycogen and an increased reliance on fat as a fuel contributing to this fatigue response [4]. Moreover, reductions in countermovement jump (CMJ) height and elevations in creatine kinase concentrations persisted $48 \mathrm{~h}$ after an English Premier League Reserve match requiring ET [3]. However, the effect of $120 \mathrm{~min}$ match-play on subjective measures of post-match recovery and subsequent performance during a 90 min competitive match requires investigation. 
Soccer players can often be exposed to congested fixture schedules, which can influence performance and injury risk [6-8]. Despite rotation of squads preventing some players from exposure to congested schedules, $\sim 40 \%$ of players complete all matches during a two or three 90 min match microcycle [7]. As transient reductions in performance have been observed during both 90 and $120 \mathrm{~min}$ matches, it is important to be able to assess measures of post-match recovery and subsequent performance during successive matches, particularly during periods of fixture congestion.

The aim of this study was twofold: (1) use GPS-derived data to assess the impact of playing a competitive match requiring ET on a competitive 90 min match played $64 \mathrm{~h}$ later; (2) elucidate the influence of ET on post-match recovery.

\section{Materials and Methods}

Four male players (two center backs, one full back, one center midfielder; age: $24 \pm 3$ years; body mass: $85.6 \pm 6.3 \mathrm{~kg}$; body fat: $9.1 \pm 0.4 \%$ (as measured by skinfold calipers); stature: $1.89 \pm 0.4 \mathrm{~m}$; $\left.V \mathrm{O}_{2 \mathrm{max}}: 61.0 \pm 1.8 \mathrm{~mL} \cdot \mathrm{kg} \cdot \mathrm{min}^{-1}\right)$ from a club playing in the third tier of English professional soccer (now in the second tier) gave informed consent to participate in this investigation. All players agreed for their data to be used for research purposes and as part of internal club procedures regarding performance and recovery assessment via written consent.

All players participated in three competitive matches in a seven-day period, with recovery measures taken $36-42 \mathrm{~h}$ post-match, prior to team training (Figure 1). Both match-days one (MD1) and three (MD3) were 90 min league matches played against opposition in the same tier, with MD1 played at the study team's home venue and MD3 played at the opposition's venue. Match-day two (MD2) was a cup match that had a duration of 120 min (i.e., included ET) played at the study team's home venue vs. a team from the first tier of English football. Players wore $10 \mathrm{~Hz}$ GPS units (Catapult MinimaxX V4, Catapult Innovations, Melbourne, Australia) during matches and training which were used to measure (presented as absolute values and per $\left.\min ^{-1}\right)$ : total and high-intensity distance covered $\left(>18 \mathrm{~km} \cdot \mathrm{h}^{-1}\right)$, number of accelerations $\left(>2 \mathrm{~m} \cdot \mathrm{s}^{-2}\right)$ and decelerations $\left(<-2 \mathrm{~m} \cdot \mathrm{s}^{-2}\right)$, and an indicator of mechanical load derived from the manufacturer (PlayerLoad ${ }^{\mathrm{TM}}$; Catapult Innovations, Melbourne, Australia). The $\mathrm{PL}_{\mathrm{Total}}$ (overall mechanical load) metric has previously been shown to possess good convergent validity with measures of exercise intensity and good test-retest reliability [9], as well as good withinand between-device reliability [10]. Furthermore, the accuracy of the MinimaxX V4 for measurement of distances covered at high speeds has been demonstrated [11]. All matches were filmed, and video footage was subsequently analyzed using a notational analysis procedure (similar to the methods of Harper et al. [1]) to assess pass and dribble accuracy (for operational definitions, see [1]).

Recovery measures included subjective measures of mood, fatigue, sleep, and muscle soreness (rated by players on a 1-7 scale ( $1=$ best, 7 = worst) using a bespoke smartphone app), which were also used to create a composite wellness score (sum of four scores divided by 28 , multiplied by 100 ). Countermovement jump (performed with hands on hips) height was measured using an electronic mat (Just Jump System, Perform Better Ltd., Warwickshire, UK).

\section{Statistical Analysis}

Data are presented as mean \pm standard deviation (SD). Magnitude-based inferences regarding the effect of ET on recovery and performance were derived utilizing methods described previously using a published spreadsheet [12]. Smallest worthwhile change was defined for each variable as 0.2 of the between-player SD. Clinical inference criteria were used to classify the effects of ET, with likelihoods classified as: most unlikely $(0-5 \%)$, very unlikely $(0.5-5 \%)$, unlikely $(5-25 \%)$, possibly likely $(25-75 \%)$, likely $(75-95 \%)$, very likely (95-99\%), most likely (>99\%). As pass and dribble accuracy were measured as a cumulative total of all four players, between-match statistical analysis was not possible. Effect sizes (ESs) were calculated using Cohen's $d$ for parametric data and $Z$ distribution values for non-parametric data, with thresholds of $0.2,0.5$, and 0.8 considered as small, medium, and large ESs, respectively [13]. 


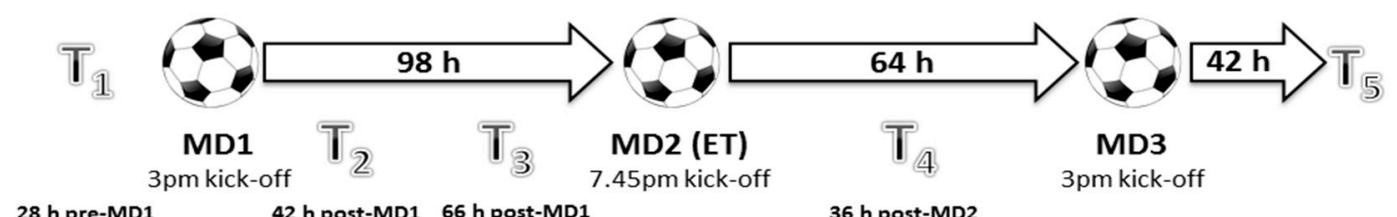

$\begin{aligned} & \text { Duration: } 80 \mathrm{~min} \\ & \text { Distance covered: } 2.8 \pm 0.3 \mathrm{~km} \\ & \text { Accelerations: } 71 \pm 4 \\ & \text { Decelerations: } 11 \pm 3 \\ & \text { Mechanical Load: } 313 \pm 64 \mathrm{AU}\end{aligned}$
$\begin{aligned} & \text { Duration: } 70 \text { min } \\ & \text { Distance covered: } 2.4 \pm 0.1 \mathrm{~km} \\ & \text { Accelerations: } 50 \pm 18 \\ & \text { Decelerations: } 12 \pm 7 \\ & \text { Mechanical Load: } 287 \pm 38 \mathrm{AU}\end{aligned}$

Figure 1. Schematic illustrating the study design. $\mathrm{MD}=$ match-day. $\mathrm{T}=$ training. Thresholds for accelerations and decelerations are $>2 \mathrm{~m} \cdot \mathrm{s}^{-2}$ and $<-2 \mathrm{~m} \cdot \mathrm{s}^{-2}$, respectively. Mechanical load derived from the manufacturer (PlayerLoad ${ }^{\mathrm{TM}}$; Catapult Innovations, Melbourne, Australia).

\section{Results}

Descriptive match performance and recovery data are presented in Tables 1 and 2 along with mean differences ( $\pm \mathrm{CL}$; confidence limits), qualitative inferences, and ESs.

Table 1. Comparison of recovery and performance variables between match-day 1 (MD1) and match-day $2(\mathrm{MD} 2$; required extra-time $(\mathrm{ET}))$. ES = effect size. CMJ = countermovement jump. $\mathrm{HI}=$ high-intensity. Mechanical load derived from the manufacturer (PlayerLoad $^{\mathrm{TM}}$; Catapult Innovations, Melbourne, Australia).

\begin{tabular}{|c|c|c|c|c|c|}
\hline & MD1 & MD2 & Mean Difference ( $\pm 90 \% \mathrm{CL})$ & Qualitative Inference & ES \\
\hline \multicolumn{6}{|c|}{ Recovery measures } \\
\hline Mood & $3 \pm 1$ & $3 \pm 1$ & 0 & N.A. & 0 \\
\hline Fatigue & $4 \pm 1$ & $5 \pm 1$ & $1 \pm 1$ & Most Likely Harmful & 2 \\
\hline Sleep & $3 \pm 1$ & $3 \pm 1$ & 0 & N.A. & 0 \\
\hline Muscle Soreness & $4 \pm 1$ & $5 \pm 1$ & $1 \pm 1$ & Very Likely Harmful & 0.9 \\
\hline Wellness Score & $50 \pm 6$ & $44 \pm 7$ & $-6 \pm 5$ & Most Likely Harmful & -0.8 \\
\hline CMJ Height (cm) & $48.5 \pm 2.3$ & $45.8 \pm 4.4$ & $-2.7 \pm 5.4$ & Very Likely Harmful & -0.6 \\
\hline \multicolumn{6}{|c|}{ Performance measures relative to match duration } \\
\hline Distance covered $\left(\mathrm{m} \cdot \mathrm{min}^{-1}\right)$ & $104.0 \pm 6.6$ & $111.5 \pm 7.3$ & $7.5 \pm 2.4$ & Most Likely Increased & 1.1 \\
\hline HI Distance $\left(\mathrm{m} \cdot \mathrm{min}^{-1}\right)$ & $4.9 \pm 1.4$ & $6.6 \pm 0.8$ & $1.7 \pm 1.2$ & Most Likely Increased & 1.3 \\
\hline Accelerations per min & $2.4 \pm 0.4$ & $3.0 \pm 0.4$ & $0.6 \pm 0.9$ & Very Likely Increased & 1.6 \\
\hline Decelerations per min & $1.0 \pm 0.2$ & $1.4 \pm 0.3$ & $0.4 \pm 0.3$ & Most Likely Increased & 2.4 \\
\hline Mechanical Load per min (AU) & $10.3 \pm 1.4$ & $11.3 \pm 1.5$ & $1.1 \pm 0.2$ & Most Likely Increased & 0.7 \\
\hline \multicolumn{6}{|c|}{ Performance measures (absolute values) } \\
\hline Distance covered $(\mathrm{km})$ & $10.4 \pm 0.6$ & $15.4 \pm 0.9$ & $4.9 \pm 0.7$ & Most Likely Increased & 7.5 \\
\hline HI distance $(\mathrm{m})$ & $438 \pm 122$ & $791 \pm 99$ & $353 \pm 100$ & Most Likely Increased & 2.9 \\
\hline Number of accelerations & $216 \pm 32$ & $358 \pm 52$ & $139 \pm 96$ & Most Likely Increased & 4.4 \\
\hline Number of decelerations & $89 \pm 16$ & $169 \pm 38$ & $80 \pm 37$ & Most Likely Increased & 5.1 \\
\hline Mechanical Load (AU) & $922 \pm 128$ & $1357 \pm 181$ & $430 \pm 70$ & Most Likely Increased & 3.4 \\
\hline Pass accuracy $(\%)$ & $88 \pm 4$ & $87 \pm 8$ & N.A. & N.A. & 0.3 \\
\hline Dribble accuracy $(\%)$ & $70 \pm 12$ & $78 \pm 12$ & N.A. & N.A. & 0.7 \\
\hline
\end{tabular}


Table 2. Comparison of recovery and performance variables between match-day 1 (MD1) and match-day 3 (MD3). CMJ = countermovement jump. $\mathrm{HI}=$ high-intensity. $\mathrm{ES}=$ effect size.

\begin{tabular}{|c|c|c|c|c|c|}
\hline & MD1 & MD3 & $\begin{array}{l}\text { Mean Difference } \\
( \pm 90 \% \mathrm{CL})\end{array}$ & Qualitative Inference & ES \\
\hline \multicolumn{6}{|c|}{ Recovery Measures } \\
\hline Fatigue & $4 \pm 1$ & $4 \pm 1$ & 0 & N.A. & 0 \\
\hline Sleep & $3 \pm 1$ & $3 \pm 1$ & 0 & N.A. & 0 \\
\hline Muscle Soreness & $4 \pm 1$ & $5 \pm 1$ & $1 \pm 1$ & Very Likely Harmful & -0.5 \\
\hline \multicolumn{6}{|c|}{ Performance measures relative to match duration } \\
\hline Distance covered $\left(\mathrm{m} \cdot \mathrm{min}^{-1}\right)$ & $104.0 \pm 6.6$ & $99.5 \pm 12.6$ & $-4.6 \pm 20$ & Unclear & 0.7 \\
\hline HI Distance $\left(\mathrm{m} \cdot \mathrm{min}^{-1}\right)$ & $4.9 \pm 1.4$ & $4.2 \pm 0.6$ & $-0.6 \pm 1.6$ & Possibly Decreased & 0.5 \\
\hline Accelerations per min & $2.4 \pm 0.4$ & $2.3 \pm 0.4$ & $-0.1 \pm 0.9$ & Unclear & 0.2 \\
\hline
\end{tabular}

\subsection{Influence of ET on Subsequent 90 Min Performance}

There was a possibly harmful effect of ET on HI distance covered during MD3 (Table 2). However, unclear effects were found for all other physical performance variables, although there were medium to large ESs for decrements in distance covered, and elevations in mechanical load (Table 2). Additionally, both pass and dribble accuracy were respectively $9.3 \%$ and $12.4 \%$ lower during MD3 vs. MD1 (Table 2).

\subsection{Influence of ET on Recovery}

Notably, post-MD2, there were very or most likely harmful effects of ET on CMJ height ( $-6 \pm 9 \%)$, muscle soreness $(+18 \pm 12 \%)$, and fatigue $(+27 \pm 4 \%)$ scores, and overall wellness score $(-13 \pm 5 \%)$ compared to post-MD1 (Table 1). Nonetheless, mood and sleep scores remained unchanged (Table 1). Furthermore, we identified very likely harmful effects on muscle soreness $(+13 \pm 14 \%)$, wellness scores $(-8 \pm 10 \%)$, and CMJ height ( $-6 \pm 9 \%)$ post-MD3 vs. post-MD1, with no differences in mood, fatigue, or sleep (Table 2).

\subsection{Individual Results}

All player's wellness scores reduced following MD2 vs. MD1 (Table 3), with each sub-variable changing negatively (i.e., increasing) or remaining the same, except for sleep for Player 2 . Countermovement jump height reduced in three out of the four players (although this was trivial for Player 2). Relative to match duration, there was an increase in performance output for all four players for each measured variable (distance covered, HI distance covered, number of accelerations and decelerations, and mechanical load) in MD2 vs. MD1, except for number of accelerations for Player 2 (Table 3).

The HI distance covered values for two players reduced from an average of 6.6 to $4.4 \mathrm{~m} \cdot \mathrm{min}^{-1}$ and 4.87 to $3.54 \mathrm{~m} \cdot \mathrm{min}^{-1}$ during MD1 vs. MD3, respectively, despite both maintaining a similar total distance covered (120 vs. 119 and 108 vs. $110 \mathrm{~m} \cdot \mathrm{min}^{-1}$, respectively). However, the HI distance covered for the other two players actually increased from an average of 3.3 to $4.0 \mathrm{~m} \cdot \mathrm{min}^{-1}$ and 4.7 to $5.0 \mathrm{~m} \cdot \mathrm{min}^{-1}$ with similar distances covered (109 vs. 114 and $122 \mathrm{vs.} 120 \mathrm{~m} \cdot \mathrm{min}^{-1}$, respectively) during MD1 vs. MD3 (Table 4). The players who had a reduction in HI distance covered during MD3 were not exposed to higher numbers of accelerations or decelerations or a higher mechanical load during MD2 than the other players, and neither player's change in subjective recovery or CMJ height was markedly different to the two other players. 
Table 3. Individual responses in recovery and performance variables between match-day 1 (MD1) and match-day 2 (MD2). Mechanical load derived from the manufacturer (PlayerLoad ${ }^{\mathrm{TM}}$; Catapult Innovations, Melbourne, Australia).

\begin{tabular}{|c|c|c|c|c|c|}
\hline & & Player 1 & Player 2 & Player 3 & Player 4 \\
\hline \multicolumn{6}{|c|}{ Recovery Measures } \\
\hline \multirow{2}{*}{ Mood } & MD1 & 4 & 2 & 3 & 3 \\
\hline & MD2 & 4 & 3 & 2 & 3 \\
\hline \multirow{2}{*}{ Fatigue } & MD1 & 4 & 3 & 4 & 4 \\
\hline & MD2 & 5 & 4 & 5 & 5 \\
\hline \multirow{2}{*}{ Sleep } & MD1 & 4 & 3 & 2 & 3 \\
\hline & MD2 & 4 & 2 & 3 & 3 \\
\hline \multirow{2}{*}{ Muscle Soreness } & MD1 & 4 & 4 & 5 & 4 \\
\hline & MD2 & 5 & 4 & 6 & 5 \\
\hline \multirow{2}{*}{ Wellness Score } & MD1 & 43 & 57 & 50 & 50 \\
\hline & MD2 & 36 & 54 & 43 & 43 \\
\hline \multirow{2}{*}{ CMJ Height (cm) } & MD1 & 50.7 & 50.3 & 46.5 & 46.6 \\
\hline & MD2 & 43.0 & 50.0 & 41.2 & 49.0 \\
\hline \multicolumn{6}{|c|}{ Performance measures relative to match duration } \\
\hline \multirow{2}{*}{ Distance covered $\left(\mathrm{m} \cdot \mathrm{min}^{-1}\right)$} & MD1 & 98.6 & 98.1 & 111.0 & 108.3 \\
\hline & MD2 & 120.2 & 120.0 & 133.5 & 135.5 \\
\hline \multirow{2}{*}{ HI Distance $\left(\mathrm{m} \cdot \mathrm{min}^{-1}\right)$} & MD1 & 3.3 & 4.9 & 4.7 & 6.6 \\
\hline & MD2 & 6.5 & 5.9 & 6.2 & 7.8 \\
\hline \multirow{2}{*}{ Accelerations per min } & MD1 & 2.6 & 2.7 & 1.9 & 2.5 \\
\hline & MD2 & 2.9 & 2.4 & 3.5 & 3.2 \\
\hline \multirow{2}{*}{ Decelerations per min } & MD1 & 0.8 & 0.9 & 1.0 & 1.2 \\
\hline & MD2 & 1.4 & 1.0 & 1.6 & 1.7 \\
\hline \multirow{2}{*}{ Mechanical Load per min (AU) } & MD1 & 9.8 & 8.6 & 12.0 & 10.6 \\
\hline & MD2 & 11.1 & 9.4 & 13.0 & 11.8 \\
\hline
\end{tabular}

Table 4. Individual responses in recovery and performance variables between match-day 1 (MD1) and match-day 3 (MD3). Mechanical load derived from the manufacturer (PlayerLoad ${ }^{\mathrm{TM}}$; Catapult Innovations, Melbourne, Australia).

\begin{tabular}{|c|c|c|c|c|c|}
\hline & & Player 1 & Player 2 & Player 3 & Player 4 \\
\hline \multicolumn{6}{|c|}{ Recovery Measures } \\
\hline \multirow[b]{2}{*}{ Mood } & MD1 & 4 & 2 & 3 & 3 \\
\hline & MD3 & 4 & 2 & 3 & 4 \\
\hline \multirow{2}{*}{ Fatigue } & MD1 & 4 & 3 & 4 & 4 \\
\hline & MD3 & 4 & 3 & 4 & 5 \\
\hline \multirow{2}{*}{ Sleep } & MD1 & 4 & 3 & 2 & 3 \\
\hline & MD3 & 4 & 3 & 2 & 3 \\
\hline \multirow{2}{*}{ Muscle Soreness } & MD1 & 4 & 4 & 5 & 4 \\
\hline & MD3 & 5 & 4 & 5 & 5 \\
\hline \multirow{2}{*}{ Wellness Score } & MD1 & 43 & 57 & 50 & 50 \\
\hline & MD3 & 49 & 57 & 50 & 39 \\
\hline \multirow{2}{*}{ CMJ Height (cm) } & MD1 & 50.7 & 50.3 & 46.5 & 46.6 \\
\hline & MD3 & 46.2 & 51.5 & 39.0 & 46.9 \\
\hline \multicolumn{6}{|c|}{ Performance measures relative to match duration } \\
\hline \multirow{2}{*}{ Distance covered $\left(\mathrm{m} \cdot \mathrm{min}^{-1}\right)$} & MD1 & 98.6 & 98.1 & 111.0 & 108.3 \\
\hline & MD3 & 105.4 & 101.2 & 81.3 & 109.9 \\
\hline \multirow{2}{*}{ HI Distance $\left(\mathrm{m} \cdot \mathrm{min}^{-1}\right)$} & MD1 & 3.3 & 4.9 & 4.7 & 6.6 \\
\hline & MD3 & 4.0 & 3.5 & 5.0 & 4.4 \\
\hline \multirow{2}{*}{ Accelerations per min } & MD1 & 2.6 & 2.7 & 1.9 & 2.5 \\
\hline & MD3 & 2 & 1.9 & 2.8 & 2.6 \\
\hline \multirow{2}{*}{ Decelerations per min } & MD1 & 0.8 & 0.9 & 1.0 & 1.2 \\
\hline & MD3 & 0.9 & 0.8 & 1.3 & 1.2 \\
\hline \multirow{2}{*}{ Mechanical Load per min (AU) } & MD1 & 9.8 & 8.6 & 12.0 & 10.6 \\
\hline & MD3 & 10.5 & 9.1 & 9.2 & 10.9 \\
\hline
\end{tabular}




\section{Discussion}

Aligning with our aims, we have demonstrated that there is compromised recovery $48 \mathrm{~h}$ following a match requiring ET in some individual players. Furthermore, performance and recovery were potentially exacerbated during, and following, a match played $64 \mathrm{~h}$ later.

We observed that players cover $\sim 5 \mathrm{~km}$ more distance, $\sim 400 \mathrm{~m}$ more HI distance, and perform an additional $\sim 170$ accelerations and $\sim 90$ decelerations during ET vs. a 90 min match (Table 1). These findings are similar to Russell et al., who used a within-match approach to analyze temporal GPS data during an English Premier League reserve cup match [3]. As ET seems to create a larger load than that observed during $90 \mathrm{~min}$, the impact of ET should be accounted for in the training and recovery of players.

We observed perturbations in ratings of fatigue, muscle soreness, overall wellness scores, and $\mathrm{CMJ}$ height $36 \mathrm{~h}$ following a match requiring ET (MD2; Tables 1 and 3). Therefore, it would seem ET has harmful effects on indices of recovery, as Russell et al. also observed decrements in CMJ performance 24 and $48 \mathrm{~h}$ following a match requiring ET, as well as elevations in concentrations of creatine kinase [3]. We observed very likely harmful effects on muscle soreness and wellness scores, and CMJ height $48 \mathrm{~h}$ post-match when comparing MD3 to MD1, including medium to large ESs for all measured recovery variables except sleep, in some of the players (Tables 2 and 4). This may indicate that recovery is exacerbated due to the extended duration of MD2. However, as shown in Tables 1 and 3, the relative intensity of MD2 was higher than MD1 (i.e., total and HI distance covered per min, the number of accelerations and decelerations per min, and mechanical load per min were all higher in MD2 vs. MD1). As such, this may have contributed to the depressed recovery response compared to MD1, rather than just match duration per se. Furthermore, the fact that the matches were played in a short period of time (i.e., congested fixture schedule) may have influenced the recovery response following MD3. Nonetheless, little data exists profiling players' perceptual recovery responses during periods of fixture congestion when all matches are $90 \mathrm{~min}$, making comparisons difficult.

We also observed a possibly harmful effect on HI distance covered during MD3; however, the effect on all other physical performance variables was unclear, with a medium ES for reductions in distance covered. At an individual level, this was particularly apparent in two of the four players. However, there was no particular variable measured during and after MD2 that may explain this change in performance in these two players. A number of factors may have contributed to this difference, including the quality of opposition faced directly in that position (i.e., central defender vs. opposition striker) or the tactics of the opposition team as a whole. Additionally, both pass and dribble accuracy were reduced during MD3 vs. MD1 (Table 2). Therefore, playing in a match requiring ET may have implications for both physical and technical performance in a subsequent $90 \mathrm{~min}$ match of close temporal proximity. Although further research is required to delineate the influence of fixture congestion per se vs. the knock-on effect of ET, no previous data has shown that fixture congestion of a similar nature (i.e., three matches in seven days) causes reductions in physical or technical performance [7]; however, we acknowledge the inferential limitations of our small dataset and sample size.

The differences in performance and recovery parameters between matches may be due to a number of factors. Firstly, readers should be cognizant of the small sample size (four professional players), and the effect this may have had on the statistical analyses of group data (i.e., inherent variation between individuals influencing the statistical outcome). Therefore, we also reported individual data for each player. The opposition in MD2 played in the first tier of English football (47 league places difference at the time of the match). We also acknowledge the influence of extraneous factors such as score-line and self-pacing strategies [14]. Moreover, MD1 was played at home and MD3 away (80 miles round trip); however, team tactics and formation were similar between matches. Furthermore, singular match-to-match comparisons can be difficult due to inherent match-play variability [15]; nonetheless, the performance and recovery data from MD1 is analogous with a $~ 4$-match average in the same group of players. There is a need for robust investigations with large datasets from multiple professional 
clubs, or the use of controlled soccer-specific protocols (i.e., [4]) with an array of recovery measures (i.e., physiological and biochemical) to further enhance knowledge of this topic.

From a practical perspective, this data suggests $120 \mathrm{~min}$ of match play causes deleterious effects on recovery and subsequent performance (in certain players), creating implications for the use of recovery modalities and training prescription following a match requiring ET. Practitioners should aim to identify players who may be particularly affected by $120 \mathrm{~min}$ of match play. With the inclusion of four substitutes instead of the traditional three being trialed and introduced in certain knockout tournaments, coaching staff can use this to substitute players whose performances and recoveries may be potentially compromised by an additional $30 \mathrm{~min}$ period.

\section{Conclusions}

Although we acknowledge the findings may reflect only the particular players involved, we conclude that ET creates a large demand on players who play the full $120 \mathrm{~min}$, creating negative corollaries of recovery. This may influence some aspects of subsequent match performance and further recovery however, this is not uniform for all players. Despite its limitations, this work provides novel findings regarding the influence of ET on recovery and subsequent match performance in professional soccer players, and is a primer for future, more expansive investigations.

Acknowledgments: The authors would like to thank the players and staff at Barnsley Football Club for their cooperation and Alex Bailey for his assistance in data acquisition.

Author Contributions: L.D.H. and N.W. conceived and designed the experiments; L.D.H. and N.W. performed the experiments; L.D.H. and N.W. analyzed the data; N.W., L.D.H., and M.R. contributed reagents/materials/analysis tools; L.D.H., N.W., R.J.N., and M.R. wrote the paper.

Conflicts of Interest: The authors declare no conflict of interest.

\section{References}

1. Harper, L.D.; West, D.J.; Stevenson, E.; Russell, M. Technical performance reduces during the extra-time period of professional soccer match-play. PLoS ONE 2014, 9, e110995. [CrossRef] [PubMed]

2. Harper, L.D.; Briggs, M.A.; McNamee, G.; West, D.J.; Kilduff, L.P.; Stevenson, E.; Russell, M. Physiological and performance effects of carbohydrate gels consumed prior to the extra-time period of prolonged simulated soccer match-play. J. Sci. Med. Sport 2015, 19, 509-514. [CrossRef] [PubMed]

3. Russell, M.; Sparkes, W.; Northeast, J.; Kilduff, L.P. Responses to a 120 min reserve team soccer match: A case study focusing on the demands of extra time. J. Sports Sci. 2015, 33, 2133-2139. [CrossRef] [PubMed]

4. Harper, L.D.; Hunter, R.; Parker, P.; Goodall, S.; Thomas, K.; Howatson, G.; West, D.J.; Stevenson, E.; Russell, M. Test-Retest Reliability of Physiological and Performance Responses to 120 Minutes of Simulated Soccer Match Play. J. Strength Cond. Res. 2016, 30, 3178-3186. [CrossRef] [PubMed]

5. Goodall, S.; Thomas, K.; Harper, L.D.; Hunter, R.; Parker, P.; Stevenson, E.; West, D.J.; Russell, M.; Howatson, G. The assessment of neuromuscular fatigue during $120 \mathrm{~min}$ of simulated soccer exercise. Eur. J. Appl. Physiol. 2017, 117, 687-697. [CrossRef] [PubMed]

6. Hägglund, M.; Waldén, M.; Magnusson, H.; Kristenson, K.; Bengtsson, H.; Ekstrand, J. Injuries affect team performance negatively in professional football: An 11-year follow-up of the UEFA Champions League injury study. Br. J. Sports Med. 2013, 47, 738-742. [CrossRef] [PubMed]

7. Carling, C.; Gregson, W.; McCall, A.; Moreira, A.; Wong del, P.; Bradley, P.S. Match running performance during fixture congestion in elite soccer: Research issues and future directions. Sports Med. 2015, 45, 605-613. [CrossRef] [PubMed]

8. Arruda, A.F.; Carling, C.; Zanetti, V.; Aoki, M.S.; Coutts, A.J.; Moreira, A. Effects of a very congested match schedule on body-load impacts, accelerations, and running measures in youth soccer players. Int. J. Sports Physiol. Perform. 2015, 10, 248-252. [CrossRef] [PubMed]

9. Barrett, S.; Midgley, A.; Lovell, R. PlayerLoad ${ }^{\mathrm{TM}}$ : Reliability, convergent validity, and influence of unit position during treadmill running. Int. J. Sports Physiol. Perform. 2014, 9, 945-952. [CrossRef] [PubMed]

10. Boyd, L.J.; Ball, K.; Aughey, R.J. The reliability of MinimaxX accelerometers for measuring physical activity in Australian football. Int. J. Sports Physiol. Perform. 2011, 6, 311-321. [CrossRef] [PubMed] 
11. Rampinini, E.; Alberti, G.; Fiorenza, M.; Riggio, M.; Sassi, R.; Borges, T.O.; Coutts, A.J. Accuracy of GPS devices for measuring high-intensity running in field-based team sports. Int. J. Sports Med. 2015, 36, 49-53. [CrossRef] [PubMed]

12. Batterham, A.M.; Hopkins, W.G. Making meaningful inferences about magnitudes. Int. J. Sports Physiol. Perform. 2006, 1, 50-57. [CrossRef] [PubMed]

13. Fritz, C.O.; Morris, P.E.; Richler, J.J. Effect size estimates: Current use, calculations, and interpretation. J. Exp. Psychol. Gen. 2012, 141, 2-18. [CrossRef] [PubMed]

14. Paul, D.J.; Bradley, P.S.; Nassis, G.P. Factors affecting match running performance of elite soccer players: Shedding some light on the complexity. Int. J. Sports Physiol. Perform. 2015, 10, 516-519. [CrossRef] [PubMed]

15. Gregson, W.; Drust, B.; Atkinson, G.; Salvo, V.D. Match-to-match variability of high-speed activities in premier league soccer. Int. J. Sports Med. 2010, 31, 237-242. [CrossRef] [PubMed]

2018 by the authors. Licensee MDPI, Basel, Switzerland. This article is an open access article distributed under the terms and conditions of the Creative Commons Attribution (CC BY) license (http://creativecommons.org/licenses/by/4.0/). 